\title{
Recurrent Ovarian Serous Adenocarcinoma
}

National Cancer Institute

\section{Source}

National Cancer Institute. Recurrent Ovarian Serous Adenocarcinoma. NCI Thesaurus.

Code C153615.

The reemergence of ovarian serous adenocarcinoma after a period of remission. 